\title{
Explorando a Motivação na Utilização de Mundos Virtuais
}

\author{
Gleizer Bierhalz Voss ${ }^{1,3}$, Evandro Franzen ${ }^{2,3}$, Magda Bercht ${ }^{3}$ \\ ${ }^{1}$ Instituto Federal Farroupilha (IFFar) \\ Rua 20 de Setembro, 2616, São Vicente do Sul - RS - Brasil \\ ${ }^{2}$ Centro Universitário Univates \\ Rua Avelino Tallini, 171, Bairro Universitário - Lajeado - RS - Brasil \\ ${ }^{3}$ Universidade Federal do Rio Grande do Sul (UFRGS) \\ Caixa Postal 15.064 - 91.501-970 - Porto Alegre - RS - Brasil \\ \{gleizer.voss,evandrofra\}@gmail.com,bercht@inf.ufrgs.br
}

\begin{abstract}
This paper presents a proposal to identify motivation from data collected during the interaction with a virtual world for teaching Physics fundamentals. Main hypothesis is that from the data collected during the interaction is possible to infer the student's motivation level. In the research developed a model was proposed based on record of actions undertaken by pupils and tables that aims to assess effort, independence and confidence demonstrated during the interaction with $V W$.
\end{abstract}

Resumo. Este artigo apresenta uma proposta para identificação da motivação a partir dos dados coletados durante a interação com um Mundo Virtual para o ensino de fundamentos de Física. A hipótese principal é que a partir dos dados coletados durante a interação é possível inferir o nível de motivação do aluno. $\mathrm{Na}$ pesquisa desenvolvida foi proposto um modelo baseado no registro das ações realizadas pelos alunos e em tabelas que visam avaliar o esforço, a independência e confiança demostrados durante a interação com o $M V$.

\section{Introdução}

O ensino de Física, é considerado difícil, pois a compreensão dos conceitos abordados necessita de experimentos com problemas concretos ou de simulações para mostrar os efeitos ou causas das noções físicas. Muitas instituições de ensino não possuem recursos financeiros ou espaços adequados para representar diversos fenômenos, que ao serem simulados qualificam o processo de ensino. Simulações computacionais podem ser utilizadas como recurso para reproduzir fenômenos, testar hipóteses, controlar e observar variáveis e situações problema (Aldrich, 2009).

Existem diversos recursos de software para realizar estas atividades de simulações, entre esses os Mundos Virtuais (MV), que se constituem em ambientes online, persistentes e gerados por computador, com os quais é possível interagir de maneira comparável ao mundo real (Bainbridge, 2010). Estratégias educacionais que propõe o uso de MV têm sido propostas para o ensino nas diversas áreas do conhecimento, entre elas a Física (Greis, Reategui e Marques, 2013; Wegener et al., 2012).

De acordo com Moran (2000), alunos curiosos e motivados facilitam enormemente o processo de ensino e aprendizagem e estimulam as melhores qualidades do professor. Os MV estimulam a participação ativa dos usuários e incentivam a resolução de problemas (Amaral, 
V Congresso Brasileiro de Informática na Educação (CBIE 2016)

Anais do XXVII Simpósio Brasileiro de Informática na Educação (SBIE 2016)

Ávila e Tarouco, 2012). O que pode contribuir para o aumento da motivação dos estudantes. Entretanto, a identificação do estado afetivo de motivação ainda é pouco explorada nestes ambientes e as iniciativas para explorar a motivação em MV são escassas.

Este trabalho aborda o uso de MV como ferramenta para investigação de aspectos associados ao comportamento dos estudantes. A hipótese principal é que a análise da interação permite verificar o engajamento, a motivação ou outros fatores relacionados ao estado afetivo do aluno. O problema a ser investigado no trabalho diz respeito a possibilidade de coletar dados da interação do usuário com o MV e a partir desses identificar padrões associados à motivação do aluno. Neste sentido, o objetivo principal é apresentar a proposta de um modelo para identificar a motivação de alunos a partir da utilização e dos dados que serão coletados durante a interação com um MV voltado ao ensino de Física.

A seção 2 descreve os fundamentos teóricos, softwares e técnicas utilizadas. Na seção 3 é apresentado o MV desenvolvido e os recursos disponíveis. O modelo proposto é apresentado na seção 4 . Na seção 5 são apresentadas as considerações finais.

\section{Mundos Virtuais e a Dimensão Afetiva}

Os Mundos Virtuais são ambientes imersivos ou metaversos, através dos quais um usuário pode realizar atividades como transitar, interagir e vivenciar experiências reais (Amaral, Ávila e Tarouco, 2012). Morgado (2009) conceitua os MV como sendo plataformas informáticas, onde podem estar telepresentes imersivamente, vários usuários, que podem se comunicar entre si. A participação desses usuários no mundo é por meio de um personagem virtual (avatar) e é através deste avatar que o usuário interage com o mundo e por ele é afetado (Morgado, 2009).

Existem diversas plataformas para a criação de Mundos Virtuais tridimensionais (3D), entre elas algumas das mais populares são o Second Life (SL), que se constitui em um aplicativo proprietário e o OpenSimulator (OpenSim), uma plataforma open source. Estas ferramentas podem ser utilizadas de várias formas no processo de ensino-aprendizagem, por exemplo, construindo cenários e objetos de aprendizagem semelhantes aos objetos reais (Figuras 1a e 1b).

A utilização de ambientes virtuais imersivos no processo de aprendizagem vem crescendo (Allison et al., 2012; Duncan, Miller e Jiang, 2012). Segundo Tarouco et al. (2012), estes recursos permitem aos alunos interagirem entre si e com o Mundo Virtual através de seus avatares. Esta interação estimula o desenvolvimento de atividades cooperativas, a partir do compartilhamento de um espaço virtual.

\subsection{OpenSimulator (OpenSim)}

O OpenSimulator (OpenSim) é um aplicativo multiplataforma de código aberto, um servidor multiusuário de aplicações 3D (Opensim, 2015). Pode ser usado para criar um ambiente virtual (ou mundo) que pode ser acessado por vários usuários (Opensim, 2015).

Tem seu código fonte distribuído sob licença Berkeley Software Distribution (BSD), permitindo que seja incorporado a produtos proprietários. Ao mesmo tempo, o OpenSim é compatível com uma série de viewers (e.g., Singularity, Firestorm, Imprudence, entre outros). 
V Congresso Brasileiro de Informática na Educação (CBIE 2016)

Anais do XXVII Simpósio Brasileiro de Informática na Educação (SBIE 2016)

\subsection{Computação Afetiva e Motivação}

A identificação automática das emoções ou das características que definem o estado de ânimo de um aluno, por exemplo, pode ser baseada em registros de ações, atividades dos usuários durante a interação dos mesmos com um sistema. Entretanto, para que esta identificação aconteça é necessário definir um conjunto de aspectos observáveis e um modelo que permita inferir o estado de ânimo a partir dos registros.

Dessa forma, as emoções ou sentimentos podem influenciar direta ou indiretamente o processo de aprendizado. De acordo com Longhi, Behar e Bercht (2009, p. 205), “a motivação é, dentre os fenômenos cognitivos associados aos afetivos, fator fundamental à aprendizagem". Desconsiderar fatores emocionais ao analisar a utilização de métodos ou técnicas de ensino pode levar a conclusões incorretas na avaliação dos resultados obtidos. Emoções podem ser caracterizadas como reações expressivas e fisiológicas, como sentimentos ou comportamentos diante de determinadas situações (Bercht, 2001). Neste contexto a afetividade é compreendida como o domínio das emoções, dos sentimentos e da capacidade de experimentar ou ter contato com as sensações.

Por sua vez, estado emocional ou estado mental afetivo diz respeito ao conjunto de características que determinam a emoção em um indivíduo, em determinado momento (Bercht, 2001). Entre os diversos estados afetivos pode-se citar frustração, interesse e a própria motivação.

Segundo Picard (1997), a Computação Afetiva é a área da computação que investiga os fundamentos e aplicações que visam capacitar os computadores para identificar, compreender e representar estados afetivos. Iepsen, Bercht e Reategui (2011) ressaltam que conferir a uma máquina recursos para identificar que um estudante encontra-se frustrado ou desanimado pode permitir a indicação de ações ou tarefas alternativas, durante a resolução de um problema.

Embora utilizados muitas vezes como sinônimos, o interesse e a motivação não são caracterizados pelo mesmo estado mental e a identificação de cada um envolve diferentes estratégias de observação e coleta de dados. Esses dados podem estar relacionados a características físicas, como pulsação, batimentos cardíacos, respiração ou à expressões faciais que podem indicar emoções. Outra forma de registro é utilizar sistemas computacionais que simulem ou capturem informações sobre o comportamento do aluno enquanto está utilizando o sistema. Estratégias mistas combinam dados oriundos de coletas automáticas com informações observadas e registradas manualmente por um professor ou pesquisador, por exemplo.

De acordo com Parellada e Rufini (2013, p. 744), “A motivação humana é um constructo interno, complexo e multideterminado. Diversas teorias elaboradas nos últimos 40 anos colocaram-se diante do desafio de compreendê-la, explicá-la e propor estratégias para intervenção, visando seu incremento." De Vicente e Pain (1998) argumentam que detectar o estado motivacional do aluno é de crucial importância, por exemplo, para a criação de Sistemas Tutores Inteligentes (STI) e que mais pesquisas são necessárias nesta área. Alguns exemplos de modelos propostos para identificar a motivação são: Attention, Relevance, Confidence and Satisfaction (ARCS) (Keller, 1987), Feedback, Instructor Techniques, Goals, and Interest (FIGI) (Robb, 2010) e Motivated Strategies for Learning Quetionnaire (MSLQ) (Pintrich et al., 1991). Além desses, tem-se o modelo Esforço (E), 
V Congresso Brasileiro de Informática na Educação (CBIE 2016)

Anais do XXVII Simpósio Brasileiro de Informática na Educação (SBIE 2016)

Independência (I) e Confiança (C) (EIC), o qual foi escolhido como base para este trabalho. Modelo esse encontrado em Bercht (2001) e aprimorado por Longhi (2011) que considera os estudos de Serres (1993), Del Soldato e Du Boulay (1995) e De Vicente e Pain (1998).

Serres (1993), citado por Bercht (2001), define que motivação está relacionada a crenças que o aluno possui, à independência e confiança demonstradas. O esforço é um indicativo de motivação intrínseca e a sua utilização na maioria dos casos é mais indicada do que outros aspectos, como o desempenho, por exemplo (Del Soldato e Du Boulay, 1995).

\subsection{Trabalhos Relacionados}

Greis, Reategui e Marques (2013) descrevem um Mundo Virtual, desenvolvido no Second Life, para simulação de fenômenos físicos como uma ferramenta para aumentar o engajamento dos estudantes no processo de aprendizagem. Foram realizadas observações e testes que visavam coletar evidências do engajamento do aluno durante a utilização do simulador.

Além da plataforma SecondLife, uma precursora na popularização dos MV, mais recentemente o OpenSim tem sido muito utilizado para o desenvolvimento de experimentos na área educacional, por exemplo, na criação de laboratórios virtuais. No trabalho desenvolvido por Tarouco et al. (2012), é apresentada a construção de um laboratório virtual para o ensino de geometria. Ainda, o desenvolvimento de um laboratório para o ensino de redes de computadores é tema do trabalho desenvolvido por Voss et al. (2014).

No campo da Computação Afetiva, o foco de diversas pesquisas tem sido o reconhecimento de estados de ânimo. Um dos trabalhos mais relevantes foi desenvolvido por Bercht (2001), nele são aplicados fundamentos da Inteligência Artificial (IA), modelando aspectos para apoiar ações de um agente de um STI. Dimensões afetivas e intelectuais são integradas para representar o modelo do aluno, o que permite ao tutor a tomada de decisões e execução de ações que levem em consideração as emoções do aluno (Bercht, 2001). A proposta descrita neste artigo, é baseada nas tabelas e nos princípios Esforço, Independência e Confiança (EIC) e propostos por Bercht (2001).

O trabalho desenvolvido por Longhi (2011) propõe utilizar técnicas de mineração de dados, em conjunto com as tabelas de Esforço, Independência e Confiança. Além da captura de dados relativos ao comportamento do aluno na interação com o sistema, dados textuais são processados com o objetivo de reconhecer a subjetividade contida nos relatos dos alunos.

Iepsen, Bercht e Reategui (2011) buscam detectar alunos que apresentam sinais de frustração no aprendizado de algoritmos. São analisadas diversas variáveis comportamentais a partir de dados coletados durante a interação dos alunos com uma ferramenta desenvolvida pelos autores. Técnicas de mineração de dados baseadas em regras são usadas para inferir sobre a frustração e associá-la a variáveis como o número de tentativas de compilação, número de erros em programas, entre outras.

Diferente dos trabalhos apresentados nesta seção, este trabalho, além de propor um ambiente computacional para apoiar o ensino de fundamentos de Física e a resolução de problemas a partir da observação de fenômenos demonstrada em simulações computacionais, também propõe um modelo para coleta de dados e a identificação de motivação do aluno a partir desses. 
V Congresso Brasileiro de Informática na Educação (CBIE 2016)

Anais do XXVII Simpósio Brasileiro de Informática na Educação (SBIE 2016)

\section{Desenvolvimento do Mundo Virtual}

Nesta seção são descritas as principais características e recursos que fazem parte do Mundo Virtual desenvolvido.

O OpenSim 0.8.1 foi escolhido por ser gratuito, open source e com uma vasta documentação no meio acadêmico. Para a visualização do MV foi selecionado o viewer Singularity, por se adequar às necessidades identificadas na construção, visualização e criação de objetos no MV. Para a hospedagem e funcionamento das ferramentas utilizadas, o Wamp Server foi escolhido por ser gratuito e conter três recursos acoplados à sua instalação (PHP, MySQL e Apache).

O Mundo Virtual desenvolvido no OpenSim consiste em um experimento que integra os seguintes componentes: vídeo introdutório, simulação Gaiola de Faraday, painel de questões de múltipla escolha (Figura 1a) e simulação Lei de Ohm, com figuras ilustrativas e calculadora de código de cores (Figura 1b), além de painéis com ajuda em formato de texto (cada questão apresenta uma alternativa de ajuda que pode ou não ser utilizada pelo aluno). $\mathrm{O}$ participante, por meio do seu avatar, interage com os elementos do $\mathrm{MV}$, podendo se deslocar entre eles de forma livre.

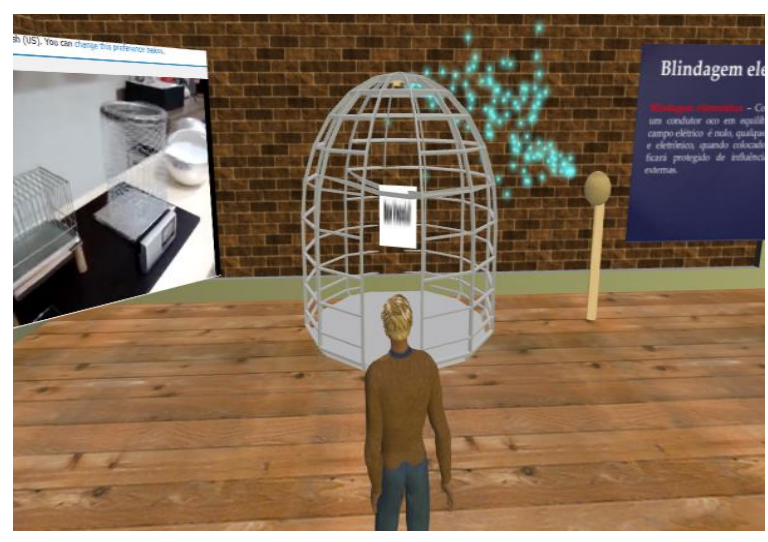

Figura 1a - Gaiola de Faraday

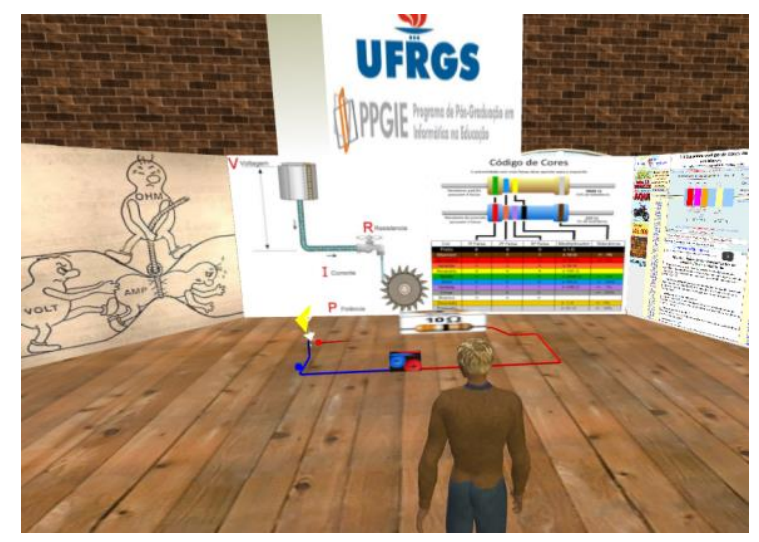

Figura 1b - Lei de Ohm

Os elementos estão inseridos no ambiente na seguinte ordem: vídeo introdutório, simulação sobre a Gaiola de Faraday, simulação sobre lei de Ohm e um painel com as questões. Ao lado do painel, estão os objetos correspondentes à ajuda para cada questão. No entanto, não há um roteiro pré-definido para os participantes, é possível assistir parte do vídeo, observar as simulações ou ir diretamente às questões. Também é possível retornar para o vídeo ou qualquer uma das simulações a qualquer momento. O conteúdo das questões que o participante irá resolver está relacionado com o vídeo e os princípios demonstrados nas simulações.

Os dados oriundos da interação dos usuários com o MV são coletados utilizando um conjunto de sensores e gravados em uma base de dados (conforme descrito na seção 4.1). São executados scripts escritos na linguagem OpenSim Script Language (OSSL) inseridos nos objetos. Ao serem clicados ou na detecção da presença do avatar, é possível armazenar uma descrição detalhada de cada etapa que ocorre durante o experimento.

A gravação ocorre por meio de dois arquivos. O primeiro é o script inserido no MV que captura as interações previamente definidas e envia para uma página PHP. Já o segundo 
V Congresso Brasileiro de Informática na Educação (CBIE 2016)

Anais do XXVII Simpósio Brasileiro de Informática na Educação (SBIE 2016)

é este arquivo PHP que recebe as informações e retorna a confirmação de gravação do registro do usuário realizando a inserção dos registros nas tabelas pré-definidas (Nunes, Voss e Cazella, 2015). Dentre os diversos dados gerados das interações dos usuários no MV, o presente estudo buscou reunir um conjunto de dados específicos para a identificação dos índices de Esforço, Independência e Confiança.

\section{Proposta para Identificação da Motivação}

A proposta para identificação da motivação é baseada em um conjunto de dados coletados durante a interação do aluno com o MV e em tabelas que definem pontuações relacionadas às ações realizadas durante a interação. São apresentadas três tabelas que correspondem à avaliação dos índices de confiança, independência (Serres, 1993) e esforço (Del Soldato e Du Boulay, 1995). Salienta-se novamente que as tabelas são uma adaptação da proposta apresentada por Bercht (2001).

\subsection{Modelo de Dados}

Conforme citado na seção 3, o aluno estará imerso no MV, assistindo vídeos, observando as simulações e respondendo as questões sobre o conteúdo. Para identificar a motivação, é necessário conhecer a trajetória do participante durante a interação, ou seja, com quais elementos ele interagiu e quando ocorreu esta interação. Além disso, as respostas para as questões se constituem em dados fundamentais para esta identificação.

Os dados serão coletados, utilizando um conjunto de sensores e serão gravados em uma base de dados de um servidor MySQL. A Figura 2 mostra o modelo Entidade Relacionamento (ER) proposto para o armazenamento dos dados.

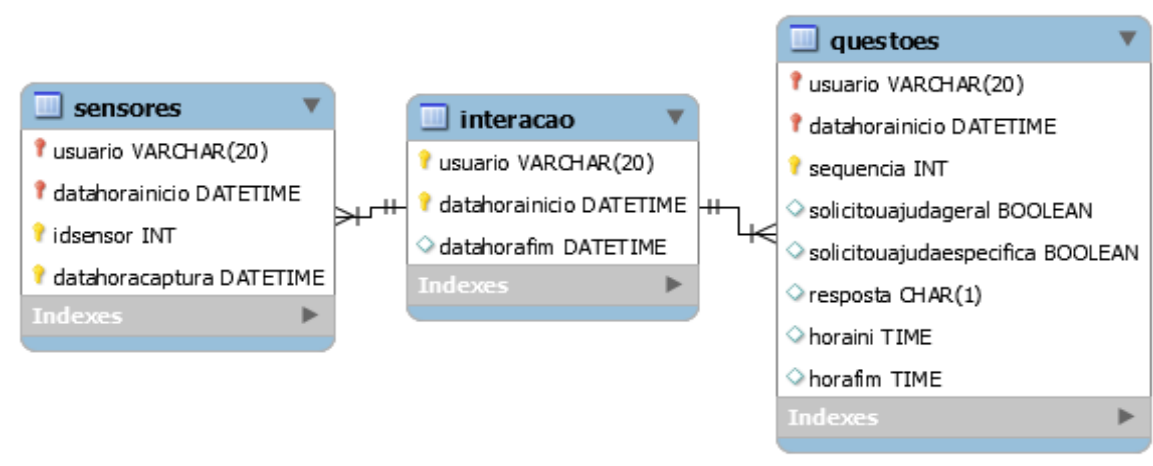

Figura 2 - Modelo ER da base de dados usada para coleta de dados

A entidade denominada "interação" registra o aluno que participou do experimento completo, além dos instantes correspondentes ao início e o fim da interação com o ambiente. Cada aluno recebe um identificador de usuário que permite o acesso e registro das informações.

A tabela "sensores" permite o registro da data e hora que o usuário passou por cada um dos sensores inseridos próximos aos objetos do MV. A partir destas informações, é possível determinar a sequência de passos e a trajetória percorrida pelo aluno. Por sua vez, em "questões" são registradas informações sobre as perguntas respondidas, incluindo as respostas e os dados sobre a utilização ou não dos recursos de ajuda disponíveis no ambiente. 
V Congresso Brasileiro de Informática na Educação (CBIE 2016)

Anais do XXVII Simpósio Brasileiro de Informática na Educação (SBIE 2016)

\subsection{Tabelas para Determinação da Motivação}

Nesta seção são apresentadas as três tabelas que definem as pontuações para o cálculo dos índices de esforço, confiança e independência. A Tabela 1 apresenta os valores para definição do esforço. O estado da tarefa é definido pelo fato do aluno ter respondido ou não cada uma das questões. Sobre cada uma das questões também se considera a utilização ou não da ajuda disponível. A tentativa de resolver a questão sem ajuda pode indicar um esforço maior neste contexto.

Tabela 1. Valores para cálculo do es forço

\begin{tabular}{lllc}
\hline Estado da tarefa & Ações & Ajuda & Fator de esforço \\
\hline Desistiu & Baixo & Com ajuda & -1 \\
Desistiu & Baixo & Sem ajuda & -2 \\
Desistiu & Médio & Com ajuda & 1 \\
Desistiu & Médio & Sem ajuda & 0 \\
Desistiu & Alto & Com ajuda & 2 \\
Desistiu & Alto & Sem ajuda & 1 \\
Realizou & Baixo & Com ajuda & 1 \\
Realizou & Baixo & Sem ajuda & 0 \\
Realizou & Médio & Com ajuda & 2 \\
Realizou & Médio & Sem ajuda & 1 \\
Realizou & Alto & Com ajuda & 3 \\
Realizou & Alto & Sem ajuda & 2 \\
\hline
\end{tabular}

Fonte: Elaborado pelos autores

Outro aspecto importante diz respeito ao conjunto de ações realizadas pelo aluno. Se um aluno assiste ao vídeo integralmente, se observa durante um tempo mínimo cada uma das simulações, é possível inferir que ele se esforçou mais para resolver as questões. A categorização das ações em alto, médio ou baixo será dada pela análise da trajetória do aluno, pelos registros dos sensores correspondentes ao vídeo e às simulações disponibilizadas no MV.

O valor máximo para o esforço é 10 pontos, mesmo que a soma da pontuação aferida em cada questão seja maior do que esta medida se considera que o aluno atingiu o nível máximo de esforço.

Tabela 2. Valores para cálculo da confiança

\begin{tabular}{llc}
\hline Situação & Estado de ajuda & Fator confiança \\
\hline Não assistiu vídeo & Não se aplica & +1 \\
Não observou simulação & Não se aplica & +1 (para cada simulação) \\
Acerta & Com ajuda & +1 \\
Acerta & Sem ajuda & +2 \\
Erra & Sem ajuda & -1 \\
Erra & Com ajuda & -2 \\
Tempo total 20\% menor que a média & Não se aplica & +2 \\
Tempo total 20\% maior que a média & Não se aplica & -2 \\
\hline
\end{tabular}

Fonte: Elaborado pelos autores 
V Congresso Brasileiro de Informática na Educação (CBIE 2016)

Anais do XXVII Simpósio Brasileiro de Informática na Educação (SBIE 2016)

A Tabela 2 descreve os fatores que permitem calcular a pontuação relacionada à confiança. Neste caso, considera-se que não ter assistido o vídeo ou observado as simulações indica uma maior confiança por parte do aluno. Além disso, considera-se o resultado da questão, acerta ou erra, tendo ou não utilizado ajuda.

O tempo total do experimento também é relevante neste caso, pois pode indicar maior confiança quando é baixo ou menor confiança nos casos em que o aluno leva um tempo maior interagindo antes de responder as questões. Para determinar este índice é calculado o tempo médio de todos os participantes em um determinado experimento e, em cada interação, é avaliado se o tempo individual é inferior ou superior à média.

A independência apresenta diversas características semelhantes à confiança, como é possível visualizar na Tabela 3. Neste caso o fato de não ter assistido o vídeo ou ter observado as simulações pode indicar maior independência, pois o participante pode acreditar que tem conhecimentos suficientes para resolver os problemas sem estas ações. Embora a falta de ações também possa ser decorrente de pouca motivação ou de uma falta de interesse, ainda assim, é considerado que há um grau de independência demonstrado pelo aluno.

Tabela 3. Valores para cálculo da independência

\begin{tabular}{llc}
\hline Estado da ajuda & Tipo da ajuda & Fator \\
\hline Não assistiu vídeo & Não se aplica & +1 \\
Não participou experiência & Não se aplica & +1 (por simulação) \\
Não utilizou e não rejeitou & Não se aplica & +1 \\
Rejeitou de forma explícita & Genérica ou específica & +2 \\
Fornecida & Genérica & -1 \\
Fornecida & Específica & -2 \\
\hline
\end{tabular}

Fonte: Elaborado pelos autores

As alternativas de ajuda disponíveis no ambiente dividem-se em genérica e específica, portanto, cada questão apresenta duas ajudas, um texto com informações gerais sobre o conteúdo e, outro mais específico que pode auxiliar mais diretamente a escolha da alternativa correta. O uso ou não do recurso e o tipo de ajuda escolhida permite inferir sobre o nível de independência do participante. Quanto menos auxílio, maior a autonomia para resolução dos problemas. Além disso, o aluno pode não fazer uso da ajuda ou informar de forma explícita que não quer o recurso, o que demonstra o seu desejo de agir de maneira autônoma.

O índice geral de motivação do aluno será dado pelos índices obtidos nos três aspectos mostrados. Embora fatores de independência e confiança sejam importantes, conforme Del Soldato e Du Boulay (1995) o esforço é, na maioria dos casos, um fator mais indicado do que outros aspectos. Por isso, a pontuação relativa ao esforço terá um peso de $50 \%$ na determinação do índice de motivação e as demais (independência e confiança) $25 \%$ cada.

\section{Considerações Finais}

A investigação sobre o uso de tecnologias para a realização de simulações e a utilização de Mundos Virtuais tem crescido e vem se consolidando como um tema relevante na educação. Entretanto, ainda existem poucos trabalhos que aliam os Mundos Virtuais à Computação Afetiva, que busca identificar ou simular emoções em sistemas computacionais. 
V Congresso Brasileiro de Informática na Educação (CBIE 2016)

Anais do XXVII Simpósio Brasileiro de Informática na Educação (SBIE 2016)

Durante a interação de um aluno com um MV é possível coletar diversos dados que podem ser usados para mostrar as ações, a sequência, o tempo e outras informações. $\mathrm{O}$ modelo que foi apresentado neste trabalho propõe a utilização dos dados coletados para identificar a motivação de quem está utilizando o ambiente.

Com a aplicação do modelo espera-se validar as tabelas propostas, a partir de uma comparação com a percepção trazida pelo autorelato do aluno e de uma análise das ações e registros sobre a interação com os recursos do ambiente. Os testes contribuirão ainda para ajustar as tabelas e caso necessário, alterar o MV para registrar de forma mais detalhada o que ocorre durante a interação.

$\mathrm{Na}$ continuidade das pesquisas serão realizados experimentos com um número maior de alunos e serão aplicadas técnicas de Mineração de Dados Educacionais (MDE) para identificar de forma automática o nível de motivação do aluno. Neste contexto os resultados obtidos com as técnicas de MDE serão comparados aos obtidos na aplicação das tabelas descritas.

\section{Referências}

ALDRICH, C. Learning Online with Games, Simulations, and Virtual Worlds. San Francisc o, CA: Jossey-Bass. 2009.

ALLISON, C., CAMPBELL, A., DAVIES, C. J., DOW, L., KENNEDY, S., MCCAFFERY, J. P. and PERERA, G. I. U. S. Growing the use of Virtual Worlds in education: an OpenSim perspective. Proceedings of the 2nd European Immersive Education Summit. 2012.

AMARAL, E., AVILA, B. G. and TAROUCO, L. M. R. Aspectos teóricos e práticos da implantação de um laboratório virtual no OpenSim. In: Anais do Simpósio Brasileiro de Informática na Educação, 2012.

BAINBRIDGE, W.S. (ed.), Online Worlds: Convergence of the Real and the Virtual, Human-Computer Interaction Series. Springer-Verlag London Limited, 2010.

BERCHT, M. Em Direção a Agentes Pedagógicos com Dimensões Afetivas. Tese de Doutorado. Instituto de Informática. UFRGS. 2001.

DE VICENTE, A. and PAIN, H. Motivation Diagnosis in Intelligent Tutoring Systems. p.8695, Springer Berlin Heidelberg. 1998.

DEL SOLDATO, T. and DU BOULAY, B. Implementation of Motivationval Tatics in Tutoring Systems. Journal of Artificial Intelligence in Education. Charlottesville. v.6, p.337-378. 1995.

DUNCAN, I., MILLER, A. and JIANG, S. A taxonomy of virtual worlds usage in education. British Journal of Educational Technology 43.6: 949-964. 2012.

GREIS, L. K., REATEGUI, E. B. and MARQUES, T. B. I.. Um Simulador de Fenômenos Físicos para Mundos Virtuais. RELATEC: Revista Latinoamericana de Tecnología Educativa, 12(1), 51-62. 2013. 
V Congresso Brasileiro de Informática na Educação (CBIE 2016)

Anais do XXVII Simpósio Brasileiro de Informática na Educação (SBIE 2016)

IEPSEN, E. F., BERCHT, M. and REATEGUI, E. Detecção e Tratamento do Estado Afetivo Frustração do Aluno na Disciplina de Algoritmos. In: Anais do Simpósio Brasileiro de Informática na Educação, 2011.

KELLER, J. M. Development and use of the ARCS model of motivational design. Journal of Instructional Development, 10(3), 2-10, 1987.

LONGHI, M. T. Mapeamento de aspectos afetivos em um ambiente virtual de aprendizagem. Tese de Doutorado. UFRGS. 2011.

LONGHI, M. T., BEHAR, P. A. and BERCHT, M. A busca pela dimensão afetiva em ambientes virtuais de aprendizagem. Em: Behar, P. (Orgs.). "Modelos pedagógicos de educação à distância”. Porto Alegre: Armed, 204-231, 2009.

MORAN, J. M. Ensino e aprendizagem inovadores com tecnologias audiovisuais e telemáticas. In: Novas Tecnologias e Mediação Pedagógica. Campinas, SP: Papirus, 2000 .

MORGADO, L. Os mundos virtuais e o ensino-aprendizagem de procedimentos. Educação \& Cultura Contemporânea, 6(13): 35-48, 2009.

NUNES, F. B., VOSS, G. B. and CAZELLA, S. C. Mineração de dados educacionais e Mundos Virtuais: um estudo exploratório no OpenSim. In Anais dos Workshops do IV Congresso Brasileiro de Informática na Educação (CBIE 2015), 2015.

OPENSIM. Página oficial da ferramenta. Disponível em: http://opensimulator.org/. Acesso em: Outubro, 2015.

PARELLADA, I. L. and RUFINI, S. É. O uso do computador como estratégia educacional: relações com a motivação e aprendizado de alunos do ensino fundamental. Psicologia: Reflexão e Crítica, v. 26, n. 4, p. 743-751, 2013.

PICARD, R. Affective Computing. Cambridge. Massachussets: MIT Press. 1997.

PINTRICH, P. R. et al. A manual for the use of the Motivated Strategies for Learning Questionnaire (MSLQ). 1991.

ROBB, C. The impact of motivational messages on student performance in community college online courses. PhD Thesis. University of Illinois, 2010.

SERRES, M. Filosofia mestiça. Rio de Janeiro. Nova Fronteira. 1993.

TAROUCO, L., ÁVILA, B., AMARAL, É., and ZEDNIK, H. VEGA-Implementando um laboratório virtual imersivo no OpenSim. Revista Novas Tecnologias na Educação, v. 10, n. 1, 2012.

VOSS, G. B., OLIVEIRA, V., NUNES, F. B., HERPICH, F., MEDINA, R. D. and BERCHT, M. Construção e Análise de um Mundo Virtual 3D para o Ensino e Aprendizagem de Redes de Computadores. In Anais do Simpósio Brasileiro de Informática na Educação, 2014

WEGENER, M.; MCINTYRE, T. J.; MCGRATH, D.; SAVAGE, C. M. and WILLIAMSON, M. Developing a virtual physics world. Australasian Journal of Educational Technology, v. 28, n. 3, p. 504-521, 2012 